\title{
Trends and predictors in all-cause and cause-specific mortality in diabetic and reference populations during 21 years of follow-up
}

\section{Niskanen, Leo}

2020-11

Niskanen , L , Partonen , T , Auvinen , A \& Haukka , J 2020 , ' Trends and predictors in all-cause and cause-specific mortality in diabetic and reference populations during 21 years of follow-up ' , Journal of Epidemiology \& Community Health , vol. 74 , no. 11 , pp. 950-956 . https://doi.org/10.1136/

http://hdl.handle.net/10138/323395

https://doi.org/10.1136/jech-2019-213602

cc_by_nc

acceptedVersion

Downloaded from Helda, University of Helsinki institutional repository.

This is an electronic reprint of the original article.

This reprint may differ from the original in pagination and typographic detail.

Please cite the original version. 


\section{Original Research Article}

\section{Trends and predictors in all-cause and cause-specific mortality in diabetic and reference populations during 21 years of follow-up}

Leo Niskanen ${ }^{1}$, Timo Partonen ${ }^{2}$, Anssi Auvinen ${ }^{3}$, Jari Haukka ${ }^{4,5}$

${ }^{1}$ Endocrinology and Diabetology, Abdominal Center, Helsinki University Hospital and Universities of Helsinki and Eastern Finland

${ }^{2}$ Department of Public Health Solutions, National Institute for Health and Welfare (THL)

${ }^{3}$ University of Tampere, Health Sciences, Faculty of Social Sciences, Finland

${ }^{4}$ Department of Public Health, University of Helsinki

${ }^{5}$ University of Tampere, Faculty of Medicine and Health Technology , Finland

Corresponding author : Jari Haukka; University of Helsinki, P.O.Box 20, FI-00014 University of Helsinki; email: jari.haukka@helsinki.fi; tel.+358504155314

Running head: Trends in mortality in diabetes population

There is no conflict of interest.

Sources of financial support: CARING study (EU grant agreement number 282526)

Data availability

The data that support the findings of this study are available from Statistics Finland, the Social Insurance Institution, and the Finnish Cancer Registry but restrictions apply to the availability of these data, which were used under license for the current study, and so are not publicly available. Data are, however, available from the authors upon reasonable request and with permission of Statistics Finland, the Social Insurance Institution, and the Finnish Cancer Registry. 
What is already known on this subject?

Patients with diabetes mellitus (DM) have a markedly higher overall mortality from coronary heart disease (CHD), as well as many other causes of death, like cancer. Recent studies indicate that mortality gap between people with diabetes and general population may have narrowed

What this study adds?

The difference between DM and reference populations in stroke mortality vanished with a later entrance to the follow-up period. There were a few differences between DM and no DM groups regarding how baseline medications were associated with mortality. The gap between the mortality of diabetic patients compared to non-diabetic subjects diminished markedly during the 21 -year period. This was driven primarily by the reduced CHD mortality. 


\section{Abstract}

\section{Background}

Patients with diabetes mellitus (DM) have a markedly higher overall mortality from coronary heart disease (CHD), as well as many other causes of death, like cancer. Since diabetes is a multisystem disease, this fact together with the increased lifespan among individuals with diabetes may also lead to the emergence of other diabetes-related complications and ultimately to diversification of the causes of death.

\section{Methods}

The study population of this observational historic cohort study consisted of DM subjects, who had purchased for at least one insulin prescription and/or one oral antidiabetic between January 1, 1997 and December 31, $2010(\mathrm{~N}=199,354)$, and a reference population matched by age, sex, and hospital district (N=199,354). Follow-up was continued until December 31, 2017. All-cause and causespecific mortality (cancer, CHD, and stroke) were analysed with Poisson and Cox's regression. Associations between baseline medications and mortality were analysed using LASSO models.

Results

The mortality rates were significantly elevated among the patients with DM. However, the relative risk of all-cause mortality between the DM and reference populations tended to converge during the follow-up. The lowering trend was most apparent in CHD mortality. The difference between DM and reference populations in stroke mortality vanished with a later entrance to the follow-up period. There were a few differences between DM and no DM groups regarding how baseline medications were associated with mortality.

\section{Conclusions}

The gap between the mortality of diabetic patients compared to non-diabetic subjects diminished markedly during the 21-year period. This was driven primarily by the reduced CHD mortality.

Keywords: cerebrovascular diseases, cancer, cohort-study, diabetes, ischaemic heart disease, mortality, pharmacoepidemiology, population 


\section{Introduction}

Patients with diabetes mellitus (DM) have markedly higher overall mortality from cardiovascular diseases (CVD) especially, but also many other causes of death, such as cancer, renal and liver diseases, infections and intentional self-harm as well as traumatic causes, which contribute to excess premature mortality [1-9]. In high-income countries, a decline in CVD mortality has been observed, but the simultaneous increase in the prevalence of diabetes, especially type 2 diabetes, implies that more patients are at an increased risk of CVDs. According to estimates of Global Burden of Disease study years of life lost (YLL) have increased globally for DM between 2006 and 2016 , with a percentage change of 25.3 [10]. On the other hand, the age-standardised rate (per $100,000)$ decreased by 2.1 during the same period.

It is conceivable that the increasing incidence of diabetes and the declining mortality indicate that the total life years spent with diabetes has increased. As diabetes is a multisystem disease, this increased life span may also lead to the emergence of other diabetes-related complications and ultimately to diversification of the causes of death. The diagnosis of type 2 diabetes markedly affects YLLs. The Emerging Risk Factors Collaboration (EFRC) reported that an average 60-yearold man with diabetes but without a history of vascular disease is about 6 years younger at the time of death than a counterpart without diabetes [7]. According to the EFRC report, $40 \%$ of YLLs due to diabetes can be attributed to nonvascular conditions. Vigorous treatment of cardiovascular risk factors and improved diabetes detection and treatment may cause time-related changes in mortality patterns and thus affect the relative causes of death and YLLs. For example, recent register data from Sweden showed that if major risk factors, like smoking, HbAlc, blood pressure, albuminuria and LDL cholesterol were under control, the risk of cardiac events approximated to a counterpart without known diabetes [11]. We analysed all-cause mortality as well as cause-specific mortality of the main causes of death in a large national cohort including all the insulin-treated patients and a random sample of half of those treated with oral antidiabetic drugs as well as a matched control group from the general population. Our data do not include information on risk factors such as blood pressure or lipid levels or smoking. Instead, we have purchases of prescribed drugs as proxies for unobserved risk factors. We analysed association between baseline drug usage and mortality, and compared predictors for DM and no DM populations. The aim of this study is to investigate trends in mortality of diabetic population compared to its reference population, and to describe factors connected to the mortality.

\section{Methods}

The study population was constructed based on the CARING Project [12] in Finland. The study population consisted of two groups. Diabetes mellitus (DM) patients included individuals who had purchased and received reimbursement for at least one insulin prescription having the Anatomical Therapeutic Chemical (ATC) code of A10A [13] and/or one oral antidiabetic (OAD) prescription (ATC code A10B) between January 1, 1997 and December 31, 2010. The reference group was individually matched to the DM group by sex, age ( \pm 1 year), region (hospital district) and start of the follow-up date. In the original population, all the insulin users in Finland in this period were included as well as a 50\% random sample of the OAD users. The size of the population was 398,708 individuals, comprising 199,354 DM reference pairs.

The study plan was approved by the Faculty of Medicine, University of Helsinki's ethical committee on January 17, 2012 (Ref 02/2012). We obtained the prescription data from the Social Insurance Institution (SII) (permission Kela 16/522/2012). Cancer data was obtained from the Finnish Cancer Registry [14], and it included the diagnosis (ICD-O-3 code)[15] and date of diagnosis (THL/264/5.05.00/2012). 
Follow-up started on the date of the first diabetes medication prescription, and ended on December 31, 2017 or on the date of death, whichever occurred first. The year 1996 was the initial wash-in period, meaning that it was guaranteed that no individual in the study population had a prescription within the 12 months before the start of the follow-up.

We obtained the information of death (the date and cause of death) and socio-economic group from Statistics Finland (permission TK-53-214-12)[16]. We used death from any cause, coronary heart disease (CHD) (ICD-10 I20-I25), stroke (I60-I69), and cancer (C00-C97) as the end-point events. Loss of follow-up was assumed to be negligibly low.

We had information on the following drug prescriptions (ATC code, date, amount of purchased medications in defined daily dose DDD) [13] from the whole study period (1996-2017): A10 (drugs used in diabetes), G03 (sex hormones and modulators of the genital system), H01 (pituitary and hypothalamic hormones and analogues), H02A (corticosteroids for systemic use, plain), H03A (thyroid preparations), H03B (antithyroid preparations), L01 (antineoplastic agents), L02 (endocrine therapy), L03 (immunostimulants), L04 (immunosuppressants), M04 (anti-gout preparations), M05 (drugs for treatment of bone diseases), N03 (anti-epileptics), N04 (anti-Parkinson's drugs), N05 (psycholeptics), and N06 (psychoanaleptics).

We categorised subjects into two groups by diabetes medication at the start of the follow-up: people with any antidiabetic medication (insulin or oral antidiabetics) and people without any DM medication. Diagnosis of cancer before the baseline (yes/no) was determined using the data from the Finnish Cancer Registry. Data on the following special reimbursement rights were obtained from the SII: severe mental disorder, chronic heart failure, hypertension, CVD, arrhythmia, ulcerative colitis.

We analysed the cohort data using conditional Poisson regression [17] models with cumulated follow-up time taken into account separately for each end-point. The following variables were used in models: diabetes group, cancer before baseline, severe mental disorder, chronic heart failure, hypertension, CVD, arrhythmia, ulcerative colitis, and socio-economic group. In conditional Poisson regression models, matching was taken into account using an "eliminate" method, thus sex, age, calendar year at the start of follow-up, and hospital district were not included in model, because matching was based on these variables. Results from the Poisson regression models are reported using mortality rate ratios (MRR).

We analysed the competing risks for four causes of death ( $\mathrm{CHD}$, stroke, cancer, other causes) using competing risks regression $[18,19]$.

Years of life lost (YLL) were calculated as the difference of the areas under the survival curves (10 year periods conditional to surviving to age 60 years, separately for men and women) comparing people with DM to the reference population. Predicted survival curves were based on Cox's proportional hazards models with the diabetes group, age, sex, year of start of follow-up, cancer before baseline, severe mental disorder, chronic heart failure, hypertension, CVD, arrhythmia, ulcerative colitis, and socio-economic group. Hospital district was used as the strata.

We studied the association between medication in the year before the start of follow-up (SOF) and mortality using LASSO (Least Absolute Shrinkage and Selection Operator) Poisson models with cumulated follow-up time as the offset [20]. The number of different medications in the whole study population (ATC codes A08, A10, C01, C02, C03, C04, C05, C07, C08, C09, C10, G03, H01, H02, H03, L01, L02, L03, L04, M04, M05, N03, N04, N05, N06, N07 were available) used in the year before SOF was 404 . We included only substances with 500 or more users in both DM and no DM populations $(\mathrm{N}=97)$ in LASSO. In addition, the following variables were always included in LASSO: age, sex, SOF year, and socio-economic group. Diabetes medications (ATC codes A10) were not included because our aim was to compare DM and no DM populations. Separate analyses were carried out for DM and no DM groups, and for men and women. The aim was to compare LASSO predictors for DM and no DM groups. The parameter $\lambda$ of LASSO was searched for by 
cross-validation and one standard error from the minimum $\lambda$ value was used [21]. Results are reported as MRRs, with a value of one meaning that the variable is not in the LASSO model. All results are reported with $95 \%$ confidence intervals. Analyses were carried out using R dataanalysis language [22] and packages Epi [23] and timereg [19].

\section{Results}

The study population consisted of 398,708 individuals (199,354 pairs), 208,538 men and 190,170 women (Table 1). The mean follow-up time was 11.3 years. Altogether, we observed 117,604 deaths, of which 30,268 were from CHD, 26,986 from cancer, and 10,129 from stroke (Table 2). The mortality rates were significantly elevated among the patients with diabetes (Appendix Figure 1 and Tables 2-4). Of note, stroke mortality was significantly higher in women than men, in unadjusted results.

Cumulative mortality curves (Appendix Figure 2) showed that proportions of the four causes of death (other, cancer, stroke, and CHD) remained about same during the follow-up in the no DM group, but in the DM group the proportion of deaths due to cancer decreased about after five years from the start of follow-up and then plateaued.

In all-cause mortality, the adjusted MRR between DM and reference populations was 1.68 (95\% confidence interval of 1.66-1.71), 1.80 (1.75-1.86) for cancer, 2.12 (2.07-2.18) for CHD, and 1.41 (1.35-1.47) for stroke mortality (Table 4). Socio-economic group had a relatively large effect on the risk of both all-cause mortality and cause-specific mortalities.

There was a considerable lowering of CHD and stroke mortality in the DM group compared to the reference population, and also a slight decrease in all-cause mortality in later start of follow-ups (SOF) (Appendix Figures 3, Figure 1). In cancer mortality, we detected no declining trend in relative risk for later SOF years.

Estimated YLLs due to DM within 15 years of the start of follow-up diminished considerably according to the calendar year of initiation of the follow-up (time when DM was detected). For a man in his 60s without cancer YLL in 15 years was 0.51 years if DM was detected in 1998 and 0.44 years if DM was detected in 2002. For a woman with the same characteristics, YLLs were 0.26 and 0.22 years, respectively (Appendix Table 1 ).

\section{LASSO models}

There were 30 substances that were included in both male and female LASSO models (Appendix Figures 4 and 5). For men, there were 37 drugs that were in the LASSO of either DM, no DM or both, and for women there were 35 drugs. The following seven drugs were only in the male models: glyceryl trinitrate (C01DA02), hydrochlorothiazide and potassium-sparing agents (C03EA01), fluocortolone (C05AA08), propranolol (C07AA05), verapamil (C08DA01), captopril (C09AA01), and candesartan (C09CA06). The following five drugs were only in the female models: enalapril (C09AA02), estradiol (G03CA03), norethisterone and estrogen (G03FA01), risedronic acid (M05BA07), and perphenazine (N05AB03).

Usage of levodopa and decarboxylase inhibitor (N04BA02) predicted the highest risk of all-cause mortality, with the risk for the DM group being considerably lower than that for the no DM group. A similar association was also observed for quetiapine (N05AH04) and risperidone (N05AX08). On the other hand, usage of prednisone (H02AB07) predicted a higher risk of all-cause mortality for the DM group. Simvastatin predicted a lower risk for all, in both men and women. Usage of estradiol (G03CA03) predicted low risk in women similarly in both groups. Interestingly, rosuvastatin (C10AA07) predicted lower mortality only in the DM group in both sexes. 


\section{Discussion}

The main finding of our study is that the difference in total mortality between the diabetic subjects and their reference population in Finland converged markedly during the 21-year period from 1997 to 2017. Thus, the main driver for the decrease in the risk rate ratios and YLLs is due to a decrease in cardiovascular mortality and its risk factors. On the other hand, a marked increase in the incidence of diabetes occurred in Finland, similar to elsewhere, thus the burden of diabetes increased in quantitative aspects [24,25]. However, in Sweden, in contrast to the results reported from many other countries, the incidence decreased modestly between 2005 and 2013, while the prevalence of pharmacologically treated diabetes increased moderately [26]. Further, during the follow-up period, a nationwide programme was on-going whose purpose was to increase the awareness of diabetes, the early identification of persons at high risk and the prevention and early treatment of type 2 diabetes [9]. Thus, the clinical diagnosis of diabetes may have occurred at an earlier phase than during the previous periods and therefore early treatment may have improved the prognosis of diabetes. To support this concept the report from recent population-based health survey indicates that the increasing incidence of diabetes has levelled off and further, the proportion of unidentified diabetes in the populations is relatively low (3.1\% in men, 1.4 in women)[24].

The reduction caused by cardiovascular disease mortality in this nationwide cohort is mainly consistent with previous reports of decreased cardiovascular mortality and risk of myocardial infarction. These findings are also relatively well in line with geographically and culturally closely related studies from Sweden. In Laxå area excess mortality in diabetic patients was very low, but they experienced less mortality reduction than reference population (Jansson et al 2010)[27]. More recent studies from Sweden show decreasing excess total and cardiovascular mortality in patients with type 2 diabetes, but noteworthy is high excess risk in younger $(<55$ years) type 2 diabetic patients $[28,29]$. This reduction in total mortality is dominated by the reduction of cardiovascular mortality and is likely due to the efficient treatment of cardiovascular risk factors, especially dyslipidaemia with statins and reductions in smoking. During era of our study, the concept of type 2 diabetes being seen as a cardiovascular risk factor emerged in clinical knowledge, and widespread campaigns to identify and treat cardiovascular risk factors in diabetes were promoted, as well as national current care guidelines emphasizing comprehensive risk assessment and active treatment. Regarding stroke mortality, the excess relative risk in diabetic patients disappeared with increasing calendar year. The reason for this finding remains obscure. In a recent Swedish study, systolic blood pressure and $\mathrm{HbA} 1 \mathrm{c}$ as well as duration of diabetes were important risk factors for stroke [11]. Since we in this study did not have access to those risk factors, the role of these in this respect remains speculative.

From non-vascular causes of death, the cancer mortality was about 50\% higher in diabetic patients than in non-diabetic subjects, accounting for one-fourth of the total mortality (Appendix Figure 2). Further, we observed that the proportion of cancer deaths decreased during the follow-up within the DM group (Appendix Figure 2). The reason for this may be that the onset of diabetes (requiring physician visits) leads to the increased detection of cancers, of which some are fatal.

Other non-vascular, non-cancer causes of death comprise a very heterogenous group. From the same cohort, we recently published the analysis of non-natural causes of death where the suicidal, traumatic and alcohol-related deaths were also higher in the diabetic than non-diabetic subjects [4]. It is also possible that some diabetic subjects were treated only with lifestyle interventions, not with medication. However, since lifestyle interventions are difficult to implement and may unnecessarily delay the start of drug treatment, the Finnish Current Care guideline (original version published in 2007 and thereafter updated several times) recommended that drug treatment with metformin 
should be initiated, if not contraindicated, concomitantly with lifestyle interventions. These guidelines have been shown to have good penetrance in primary care [30], and therefore it is likely that those clinically diagnosed with diabetes were included in the study.

Analyses of baseline medications revealed some difference in risk between DM and no DM groups. We found that DM users of quetiapine, risperidone and digoxin had a lower relative risk compared to users with no DM that is in line with observation that in primary care, structured diabetes care was highly effective among diabetic patients with co-occurring psychiatric illness [31]. Both quetiapine and risperidone are widely used antipsychotics, and the increased all-cause relative mortality among the no DM group using these antipsychotics may indicate that those patients with co-morbid DM and psychotic disorder were better taken care of compared to the reference population with psychotic disorder.

Concerning sex differences, propranol was associated with a higher risk in men in the DM group, whereas valproic acid and mirtazapine were associated with a higher risk in women in the DM group. Interestingly, estrogen users in the DM group were at a lower risk. However, valproic acid is widely prescribed not only for the treatment of epilepsy but also for bipolar disorder, and mirtazapine not only for depressive disorder but also for insomnia (although as off-label). These associations with medications may be due to the effect of the drug substance, or due to the indication of the drug. In the latter case, a drug is a proxy for the condition it was prescribed for. Or, there may be a selective prescription practice for the DM group. However, observations of risk differences are worth further study in order to detect reasons for them.

This register-based cohort study has other limitations as well. We did not have access to risk factors like body mass index, LDL cholesterol, blood pressure levels and smoking, exercise habit nor to diabetic complications. Differences in these may have partly explained differences in mortality between no-DM and DM groups. Another crucial limitation is that we used antidiabetic medication use as indicator for DM. Inclusion of diabetic individuals into no-DM group may attenuate the difference between groups, because individuals with diabetes without treatment are in higher risks.

Better care for diabetic patients may be cause for decreasing gap in mortality between diabetic population and general population. Well organized and intensive case management provides individuals with diabetes good access to preventive health care, which leads to lower mortality in due to main preventable causes of death like cardiovascular diseases and stroke. Results of Swedish study [11], where patients with diabetes and well-managed risk factors were compared with a reference group of people free from diabetes, but with risk factors as they appear in the general population are similar to this study. Results of Swedish study may also be partly due to same cause, risk factors of reference population were not measured, and in reality, risk factors of reference population may have been under estimated.

In conclusion, our study shows that a difference in cardiovascular mortality between the diabetic subjects and their reference population in Finland has converged markedly in the 21-year period of 1997 to 2010 . However, during the same period, the difference in cancer mortality between patients with DM and their reference population has increased. During the study period, YLLs due to diabetes has decreased in both men and women.

To conclude, the pattern of excess mortality in diabetic subjects is changing and this has preventive and clinical implications. 
All the authors have made substantial contributions: conception and design (LN, AA, TP, and JH), data acquisition and analysis (JH, AA), reporting and interpretation (LN, AA, TP, and JH). They all participated in drafting the article or revising it critically for important intellectual content, and all have approved the final version.

\section{Declaration of interests}

We declare no competing interests.

\section{Acknowledgement}

We would like to thank the CARING study (European Commission, Seventh Framework Programme grant agreement number 282526) group for co-operation during the project [32] that made these data available to the authors (AA and JH are members of the CARING group).

\section{Data availability}

The data that support the findings of this study are available from Statistics Finland, the Social Insurance Institution, and the Finnish Cancer Registry but restrictions apply to the availability of these data, which were used under license for the current study, and so are not publicly available. Data are, however, available from the authors upon reasonable request and with permission of Statistics Finland, the Social Insurance Institution, and the Finnish Cancer Registry. 
1 Webb RT, Lichtenstein P, Dahlin M, et al. Unnatural Deaths in a National Cohort of People Diagnosed With Diabetes. Diabetes Care 2014;37:2276-83. doi:10.2337/dc14-0005 2 Cheng YJ, Imperatore G, Geiss LS, et al. Trends and Disparities in Cardiovascular Mortality Among U.S. Adults With and Without Self-Reported Diabetes, 1988-2015. Diabetes Care 2018;41:2306-15. doi:10.2337/dc18-0831

3 Gregg EW, Cheng YJ, Srinivasan M, et al. Trends in cause-specific mortality among adults with and without diagnosed diabetes in the USA: an epidemiological analysis of linked national survey and vital statistics data. The Lancet Published Online First: May 2018. doi:10.1016/S0140-6736(18)30314-3

$4 \quad$ Niskanen L, Partonen T, Auvinen A, et al. Excess mortality in Finnish diabetic subjects due to alcohol, accidents and suicide: a nationwide study. European Journal of Endocrinology 2018;179:299-306. doi:10.1530/EJE-18-0351

$5 \quad$ Haukka J, Niskanen L, Auvinen A. Risk of cause-specific death in individuals with cancer - modifying role diabetes, statins, and metformin. International Journal of Cancer Published Online First: August 2017. doi:10.1002/ijc.31016

6 Harding JL, Pavkov ME, Magliano DJ, et al. Global trends in diabetes complications: a review of current evidence. Diabetologia Published Online First: 31 August 2018. doi:10.1007/s00125-018-4711-2

$7 \quad$ Angelantonio ED, Kaptoge S, Wormser D, et al. Association of Cardiometabolic Multimorbidity With Mortality. JAMA 2015;314:52-60. doi:10.1001/jama.2015.7008

$8 \quad$ Seshasai SRK, Kaptoge S, Thompson A, et al. Diabetes mellitus, fasting glucose, and risk of cause-specific death. $N$ Engl J Med 2011;364:829-41. doi:10.1056/NEJMoa1008862

9 Saaristo T, Moilanen L, Korpi-Hyövälti E, et al. Lifestyle Intervention for Prevention of Type 2 Diabetes in Primary Health Care: One-year follow-up of the Finnish National Diabetes Prevention Program (FIN-D2D). Diabetes Care 2010;33:2146-51. doi:10.2337/dc10-0410 10 Global, regional, and national age-sex specific mortality for 264 causes of death, 1980-2016: a systematic analysis for the Global Burden of Disease Study 2016. The Lancet 2017;390:1151-210. doi:10.1016/S0140-6736(17)32152-9

11 Rawshani A, Rawshani A, Franzén S, et al. Risk Factors, Mortality, and Cardiovascular Outcomes in Patients with Type 2 Diabetes. New England Journal of Medicine 2018;379:633-44. doi:10.1056/NEJMoa1800256

12 CARING CAncer Risk and INsulin analoGues Common Study Protocol.

2013.http:/www.encepp.eu/encepp/openAttachment/fullProtocol/5386

13 Anonym. ATC/DDD Index 2006. WHO Collaborating Centre for Drug Statistics Methodology 2006. http://www.whocc.no/atcddd/

14 Finnish Cancer Registry. Register description of Finnish Cancer Registry. http://www.cancer.fi/syoparekisteri/en/registration/register-description/ (accessed 9 Feb 2016). $15 \quad$ WHO $\mid$ International Classification of Diseases for Oncology, 3rd Edition (ICD-O-3). http://www.who.int/classifications/icd/adaptations/oncology/en/ (accessed 25 Feb 2014).

16 Lahti RA, Penttilä A. The validity of death certificates: routine validation of death certification and its effects on mortality statistics. Forensic Science International 2001;115:15-32. doi:10.1016/S0379-0738(00)00300-5

17 Turner H, Firth D. Generalized nonlinear models in R: An overview of the gnm package. 2018 . https://cran.r-project.org/package=gnm

18 Scheike TH, Sun Y, Zhang M-J, et al. A semiparametric random effects model for multivariate competing risks data. Biometrika 2010;97:133-45. doi:10.1093/biomet/asp082 19 Scheike TH, Zhang M-J. Analyzing competing risk data using the R timereg package. Journal of statistical software 2011;38.http://www.ncbi.nlm.nih.gov/pmc/articles/PMC3375021/ 
(accessed 31 May 2016).

20 Tibshirani R. Regression Shrinkage and Selection via the Lasso. Journal of the Royal Statistical Society Series B (Methodological) 1996;58:267-88.

21 Friedman JH, Hastie T, Tibshirani R. Regularization Paths for Generalized Linear Models via Coordinate Descent. Journal of Statistical Software 2010;33:1-22.

doi:10.18637/jss.v033.i01

$22 \quad \mathrm{R}$ Core Team. R: A Language and Environment for Statistical Computing. Vienna, Austria: : R Foundation for Statistical Computing 2016. https:/www.R-project.org/

23 Carstensen B, Plummer M, Laara E, et al. Epi: A package for statistical analysis in epidemiology. 2008. http://www.pubhealth.ku.dk/ bxc/Epi/

$24 \quad$ Koponen $\mathrm{P}$, Borodulin K, Lundqvist A, et al. Terveys, toimintakyky ja hyvinvointi Suomessa FinTerveys 2017-tutkimus. Helsinki, Finland: 2018. http://urn.fi/URN:ISBN:978-952$343-105-8$

25 FinHealth 2017 Study - Methods. Helsinki, Finland: : Finnish Institute for Health and Welfare 2019. http://urn.fi/URN:ISBN:978-952-343-449-3

26 Jansson SPO, Fall K, Brus O, et al. Prevalence and incidence of diabetes mellitus: a nationwide population-based pharmaco-epidemiological study in Sweden. Diabetic Medicine 2015;32:1319-28. doi:10.1111/dme.12716

27 Jansson SPO, Andersson DKG, Svärdsudd K. Mortality Trends in Subjects With and Without Diabetes During 33 Years of Follow-up. Diabetes Care 2010;33:551-6. doi:10.2337/dc090680

28 Andersson T, Hjerpe P, Carlsson AC, et al. Mortality trends and cause of death in patients with new-onset type 2 diabetes and controls: A 24-year follow-up prospective cohort study. Diabetes Research and Clinical Practice 2018;138:81-9. doi:10.1016/j.diabres.2018.01.038

29 Tancredi M, Rosengren A, Svensson A-M, et al. Excess Mortality among Persons with Type 2 Diabetes. New England Journal of Medicine 2015;373:1720-32. doi:10.1056/NEJMoa1504347

30 Niskanen L, Hahl J, Haukka J, et al. Type 2 diabetes and treatment intensification in primary care in Finland. Acta Diabetologica Published Online First: 6 August 2018. doi:10.1007/s00592-018-1199-7

31 Larsen JR, Siersma VD, Davidsen AS, et al. The excess mortality of patients with diabetes and concurrent psychiatric illness is markedly reduced by structured personal diabetes care: A 19-year follow up of the randomized controlled study Diabetes Care in General Practice (DCGP). General Hospital Psychiatry 2016;38:42-52. doi:10.1016/j.genhosppsych.2015.10.001

32 General introduction | CARING. http://www.caring-diabetes.eu/ (accessed 23 Apr 2015). 
Table 1 Basic characteristics of study population at start of follow-up, frequencies (\%).

\begin{tabular}{|c|c|c|c|}
\hline & & No DM & DM \\
\hline All & & 199354 & 199354 \\
\hline \multirow[t]{2}{*}{ Sex } & Male & $\begin{array}{r}104269 \\
(52.3)\end{array}$ & $\begin{array}{r}104269 \\
(52.3)\end{array}$ \\
\hline & Female & $95085(47.7)$ & $95085(47.7)$ \\
\hline \multirow[t]{3}{*}{ Age } & $0-30$ & $14841(7.4)$ & $14841(7.4)$ \\
\hline & $30-60$ & $84326(42.3)$ & $84326(42.3)$ \\
\hline & over 60 & $\begin{array}{r}100187 \\
(50.3)\end{array}$ & $\begin{array}{r}100187 \\
(50.3)\end{array}$ \\
\hline \multirow[t]{3}{*}{ Start of follow-up } & 1997-2001 & $48850(24.5)$ & $48850(24.5)$ \\
\hline & $2002-2006$ & $63617(31.9)$ & 63617 (31.9) \\
\hline & 2007-2011 & $86887(43.6)$ & 86887 (43.6) \\
\hline Cancer & & $5307(2.7)$ & $7703(3.9)$ \\
\hline Severe mental disorder & & $4713(2.4)$ & $8721(4.4)$ \\
\hline Chronic heart failure & & $4308(2.2)$ & $8809(4.4)$ \\
\hline Hypertension & & $32514(16.3)$ & $59564(29.9)$ \\
\hline Cardiovascular disease & & $14270(7.2)$ & $21211(10.6)$ \\
\hline Arrythmia & & $3376(1.7)$ & $5003(2.5)$ \\
\hline Ulcerative colitis & & $1085(0.5)$ & $1277(0.6)$ \\
\hline \multirow[t]{9}{*}{ Socio-economic group } & $\begin{array}{l}\text { Upper-level } \\
\text { employees }\end{array}$ & $18521(9.3)$ & $13657(6.9)$ \\
\hline & $\begin{array}{l}\text { Self-employed } \\
\text { persons }\end{array}$ & $4302(2.2)$ & $3467(1.7)$ \\
\hline & Farmer & $8762(4.4)$ & $8040(4.0)$ \\
\hline & $\begin{array}{l}\text { Lower-level } \\
\text { employees }\end{array}$ & $27747(13.9)$ & $24070(12.1)$ \\
\hline & Manual workers & $29579(14.8)$ & $29051(14.6)$ \\
\hline & Students & $3588(1.8)$ & $3611(1.8)$ \\
\hline & Pensioners & $88304(44.3)$ & $96200(48.3)$ \\
\hline & Others & $13578(6.8)$ & $15411(7.7)$ \\
\hline & Unknown & $4973(2.5)$ & $5847(2.9)$ \\
\hline
\end{tabular}


Table 2. Number of deaths and all-cause mortality rates (per 1000 person-years) with $95 \%$ confidence intervals for baseline characteristics.

\begin{tabular}{|c|c|c|c|c|c|c|c|}
\hline & & \multicolumn{3}{|c|}{ No DM } & \multicolumn{3}{|r|}{ DM } \\
\hline & & P-years & Events & Rate (95\% CI) & $\begin{array}{l}\text { P- } \\
\text { years }\end{array}$ & Events & Rate (95\% CI) \\
\hline \multirow[t]{2}{*}{ Sex } & Male & 1219 & 25925 & $21.26(21.01-21.52)$ & 1119 & 36943 & $33.01(32.67-33.35)$ \\
\hline & Female & 1113 & 23702 & $21.29(21.02-21.56)$ & 1045 & 31034 & $29.70(29.37-30.03)$ \\
\hline \multirow[t]{3}{*}{ Age } & $0-30$ & 198 & 98 & $0.49(0.40-0.60)$ & 197 & 301 & $1.53(1.36-1.71)$ \\
\hline & $30-60$ & 1088 & 6987 & $6.42(6.27-6.57)$ & 1024 & 15331 & $14.97(14.74-15.21)$ \\
\hline & over 60 & 1046 & 42542 & $40.67(40.28-41.06)$ & 943 & 52345 & $55.49(55.02-55.97)$ \\
\hline \multirow[t]{3}{*}{ Start of follow-up } & $1997-2001$ & 771 & 18770 & $24.35(24.00-24.70)$ & 687 & 25730 & $37.47(37.01-37.93)$ \\
\hline & $2002-2006$ & 806 & 16988 & $21.08(20.77-21.40)$ & 747 & 23783 & $31.83(31.42-32.23)$ \\
\hline & $2007-2011$ & 756 & 13869 & $18.34(18.04-18.65)$ & 730 & 18464 & $25.29(24.93-25.66)$ \\
\hline \multirow[t]{2}{*}{ Cancer } & no & 2287 & 46809 & $20.47(20.29-20.66)$ & 2112 & 62788 & $29.73(29.50-29.96)$ \\
\hline & yes & 46 & 2818 & $61.25(59.01-63.55)$ & 52 & 5189 & $99.74(97.05-102.49)$ \\
\hline \multirow[t]{2}{*}{ Severe mental disorder } & no & 2285 & 47629 & $20.85(20.66-21.03)$ & 2074 & 64571 & $31.13(30.90-31.38)$ \\
\hline & yes & 48 & 1998 & $41.76(39.95-43.63)$ & 90 & 3406 & $37.79(36.54-39.08)$ \\
\hline \multirow[t]{2}{*}{ Chronic heart failure } & no & 2299 & 46216 & $20.11(19.92-20.29)$ & 2098 & 60832 & $29.00(28.77-29.23)$ \\
\hline & yes & 34 & 3411 & $100.33(96.99-103.76)$ & 67 & 7145 & $\begin{array}{r}107.38(104.90- \\
109.90)\end{array}$ \\
\hline \multirow[t]{2}{*}{ Hypertension } & no & 1984 & 36069 & $18.18(17.99-18.37)$ & 1539 & 42421 & $27.56(27.30-27.82)$ \\
\hline & yes & 348 & 13558 & $38.92(38.27-39.59)$ & 625 & 25556 & $40.91(40.41-41.42)$ \\
\hline \multirow[t]{2}{*}{ Cardiovascular disease } & no & 2194 & 41685 & $19.00(18.82-19.18)$ & 1970 & 55588 & $28.22(27.98-28.45)$ \\
\hline & yes & 139 & 7942 & $57.26(56.01-58.54)$ & 194 & 12389 & $63.87(62.75-65.01)$ \\
\hline \multirow[t]{2}{*}{ Arrythmia } & no & 2301 & 47694 & $20.73(20.54-20.91)$ & 2122 & 64687 & $30.49(30.25-30.72)$ \\
\hline & yes & 32 & 1933 & $61.26(58.56-64.06)$ & 42 & 3290 & $77.80(75.16-80.50)$ \\
\hline Ulcerative colitis & no & 2321 & 49406 & $21.29(21.10-21.48)$ & 2151 & 67590 & $31.43(31.19-31.67)$ \\
\hline
\end{tabular}




\begin{tabular}{|l|l|r|r|r|r|r|r|}
\hline & yes & 12 & 221 & $18.45(16.10-21.05)$ & 13 & 387 & $28.90(26.09-31.93)$ \\
\hline Socio-economic group & Upper-level employees & 235 & 856 & $3.64(3.40-3.89)$ & 167 & 1428 & $8.54(8.10-9.00)$ \\
\hline & Self-employed persons & 59 & 381 & $6.49(5.86-7.18)$ & 46 & 641 & $13.97(12.91-15.10)$ \\
\hline & Farmer & 112 & 625 & $5.57(5.14-6.03)$ & 99 & 1328 & $13.40(12.69-14.14)$ \\
\hline & Lower-level employees & 351 & 1317 & $3.75(3.55-3.96)$ & 294 & 2381 & $8.09(7.77-8.43)$ \\
\hline & Manual workers & 380 & 2383 & $6.28(6.03-6.53)$ & 352 & 4365 & $12.39(12.03-12.77)$ \\
\hline & Students & 47 & 118 & $2.52(2.09-3.02)$ & 46 & 247 & $5.41(4.76-6.13)$ \\
\hline & Pensioners & 914 & 41141 & $45.03(44.59-45.46)$ & 912 & 52177 & $57.20(56.71-57.70)$ \\
\hline & Others & 176 & 2023 & $11.51(11.02-12.03)$ & 186 & 3702 & $19.89(19.25-20.54)$ \\
\hline & Unknown & 59 & 783 & $13.17(12.26-14.13)$ & 62 & 1708 & $27.72(26.42-29.06)$ \\
\hline
\end{tabular}


Table 3. Cause-specific death and mortality rates per 1000 person-years.

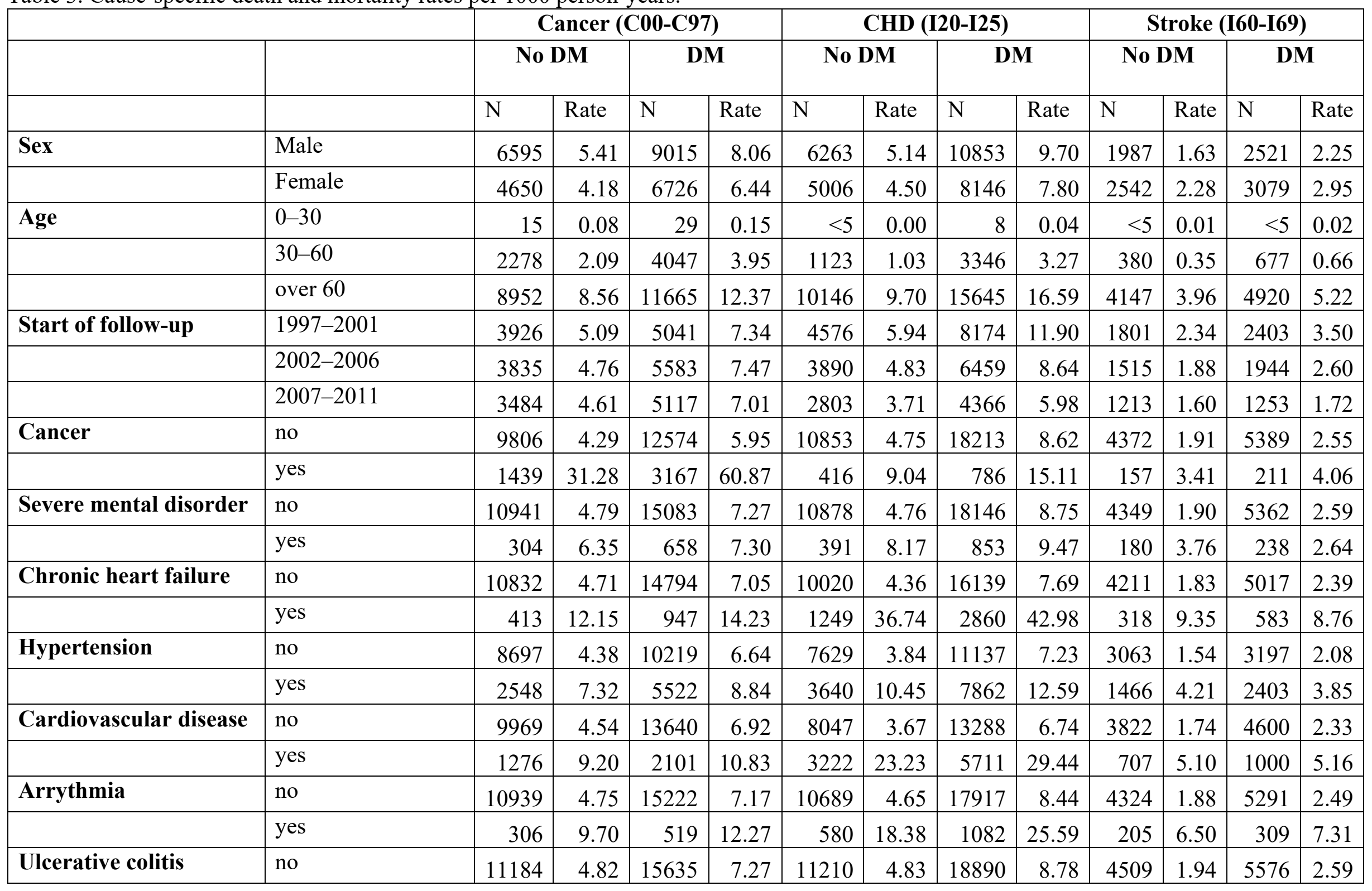




\begin{tabular}{|c|c|c|c|c|c|c|c|c|c|c|c|c|c|}
\hline & yes & 61 & 5.09 & 106 & 7.92 & 59 & 4.93 & 109 & 8.14 & 20 & 1.67 & 24 & 1.79 \\
\hline \multirow[t]{9}{*}{ Socio-economic group } & $\begin{array}{l}\text { Upper-level } \\
\text { employees }\end{array}$ & 385 & 1.64 & 499 & 2.98 & 126 & 0.54 & 290 & 1.73 & 40 & 0.17 & 67 & 0.40 \\
\hline & $\begin{array}{l}\text { Self-employed } \\
\text { persons }\end{array}$ & 144 & 2.45 & 176 & 3.84 & 66 & 1.12 & 162 & 3.53 & 27 & 0.46 & 48 & 1.05 \\
\hline & Farmer & 245 & 2.18 & 425 & 4.29 & 110 & 0.98 & 313 & 3.16 & 41 & 0.37 & 78 & 0.79 \\
\hline & $\begin{array}{l}\text { Lower-level } \\
\text { employees }\end{array}$ & 599 & 1.71 & 919 & 3.12 & 157 & 0.45 & 416 & 1.41 & 74 & 0.21 & 91 & 0.31 \\
\hline & Manual workers & 889 & 2.34 & 1344 & 3.82 & 442 & 1.16 & 1022 & 2.90 & 102 & 0.27 & 206 & 0.58 \\
\hline & Students & 30 & 0.64 & 61 & 1.34 & 22 & 0.47 & 44 & 0.96 & 6 & 0.13 & 12 & 0.26 \\
\hline & Pensioners & 8158 & 8.93 & 10901 & 11.95 & 9853 & 10.78 & 15563 & 17.06 & 4063 & 4.45 & 4821 & 5.29 \\
\hline & Others & 597 & 3.40 & 888 & 4.77 & 368 & 2.09 & 807 & 4.34 & 114 & 0.65 & 172 & 0.92 \\
\hline & Unknown & 198 & 3.33 & 528 & 8.57 & 125 & 2.10 & 382 & 6.20 & 62 & 1.04 & 105 & 1.70 \\
\hline
\end{tabular}


Table 4 Mortality rate ratios ( $95 \%$ confidence intervals) based on conditional Poisson model for sex, age, and hospital district matched cohort design.

\begin{tabular}{|c|c|c|c|c|c|}
\hline & & All-cause & Cancer & CHD & Stroke \\
\hline & & \multicolumn{4}{|c|}{ Univariate } \\
\hline \multirow[t]{2}{*}{ DM vs no DM } & & $1.88(1.85-1.90)$ & $2.15(2.09-2.21)$ & $2.47(2.40-2.53)$ & $1.52(1.46-1.59)$ \\
\hline & & \multicolumn{4}{|c|}{ Multivariate } \\
\hline DM vs no DM & & $1.68(1.66-1.71)$ & $1.80(1.75-1.86)$ & $2.12(2.07-2.18)$ & $1.41(1.35-1.47)$ \\
\hline Cancer vs no & & $3.33(3.19-3.48)$ & $30.84(28.32-33.58)$ & $0.90(0.82-0.99)$ & $0.83(0.71-0.98)$ \\
\hline Severe mental disorder vs no & & $1.99(1.89-2.10)$ & $1.29(1.15-1.45)$ & $1.78(1.60-1.98)$ & $1.75(1.47-2.07)$ \\
\hline Chronic heart failure vs no & & $1.49(1.44-1.55)$ & $1.17(1.06-1.29)$ & $1.89(1.77-2.03)$ & $1.15(1.02-1.30)$ \\
\hline Hypertension vs no & & $1.15(1.12-1.17)$ & $0.98(0.94-1.02)$ & $1.36(1.31-1.41)$ & $1.41(1.32-1.51)$ \\
\hline Cardiovascular disease vs no & & $1.18(1.15-1.21)$ & $0.84(0.79-0.90)$ & $2.58(2.46-2.71)$ & $1.07(0.98-1.16)$ \\
\hline Arrythmia vs no & & $1.15(1.09-1.21)$ & $0.87(0.78-0.98)$ & $1.11(1.01-1.22)$ & $1.39(1.19-1.63)$ \\
\hline Ulcerative colitis vs no & & $1.00(0.88-1.14)$ & $1.03(0.79-1.34)$ & $1.13(0.86-1.48)$ & $1.03(0.64-1.66)$ \\
\hline \multirow{6}{*}{$\begin{array}{l}\text { Socio-economic group vs upper - } \\
\text { level employees }\end{array}$} & Self-employed persons & $1.46(1.31-1.63)$ & $1.07(0.88-1.30)$ & $1.24(0.98-1.57)$ & $2.04(1.34-3.10)$ \\
\hline & Farmer & $1.48(1.36-1.62)$ & $1.18(1.01-1.39)$ & $1.65(1.35-2.01)$ & $1.97(1.36-2.86)$ \\
\hline & Lower-level employees & $1.25(1.16-1.35)$ & $1.25(1.10-1.42)$ & $1.09(0.91-1.30)$ & $1.40(1.00-1.95)$ \\
\hline & Manual workers & $1.86(1.74-1.99)$ & $1.67(1.48-1.89)$ & $1.93(1.65-2.25)$ & $1.70(1.25-2.30)$ \\
\hline & Students & $2.39(1.99-2.87)$ & $1.86(1.30-2.64)$ & $3.79(2.38-6.04)$ & $3.30(1.43-7.57)$ \\
\hline & Pensioners & $3.92(3.67-4.18)$ & $2.07(1.84-2.33)$ & $3.36(2.90-3.91)$ & $4.28(3.22-5.69)$ \\
\hline
\end{tabular}




\begin{tabular}{|l|l|r|r|r|r|}
\hline & Others & $3.75(3.49-4.04)$ & $2.09(1.83-2.39)$ & $3.41(2.88-4.02)$ & $3.11(2.27-4.26)$ \\
\hline & Unknown & $10.07(9.13-11.11)$ & $4.83(4.04-5.78)$ & $7.68(6.18-9.52)$ & $13.69(9.10-20.60)$ \\
\hline
\end{tabular}




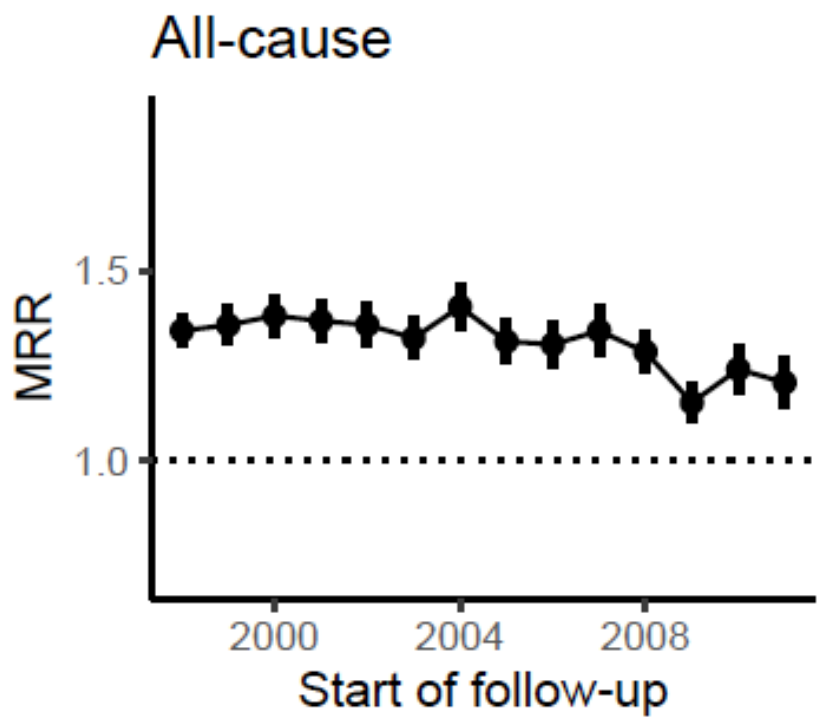

$\mathrm{CHD}$

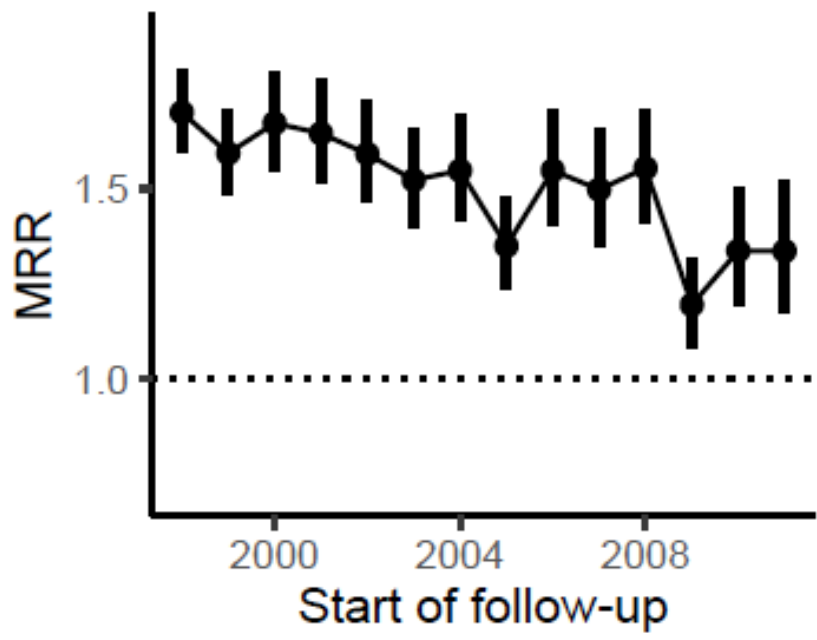

Cancer

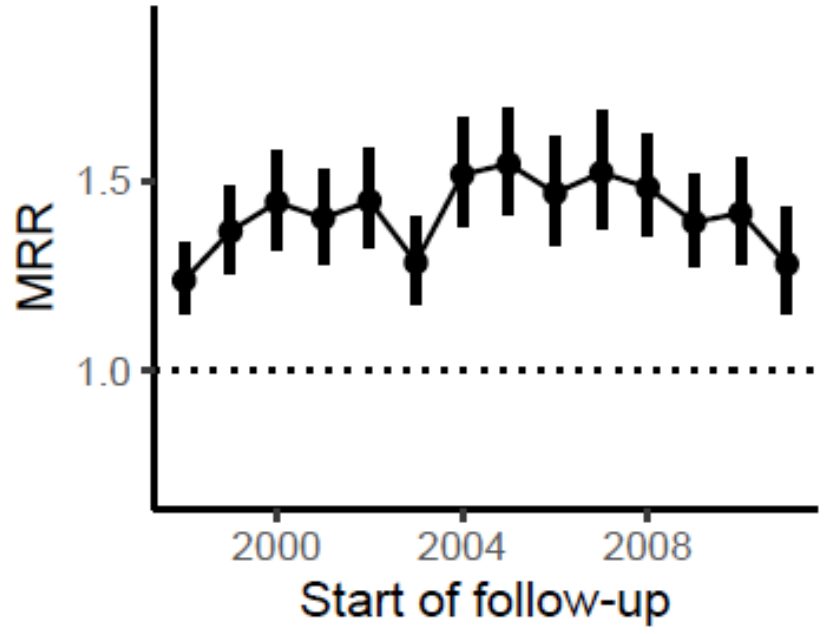

Stroke

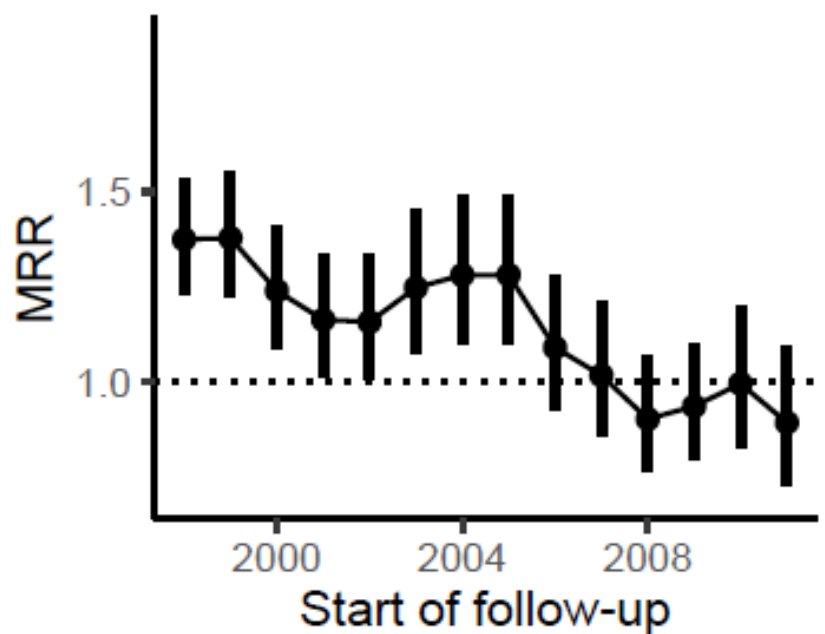

Figure 1. Mortality rate ratios with $95 \%$ confidence intervals for comparing DM to no DM. Results from Poisson regression models adjusted for sex, age, start of follow-up year, hospital district, socioeconomic group, special reimbursement rights and interaction between DM and start of follow-up year in model. 


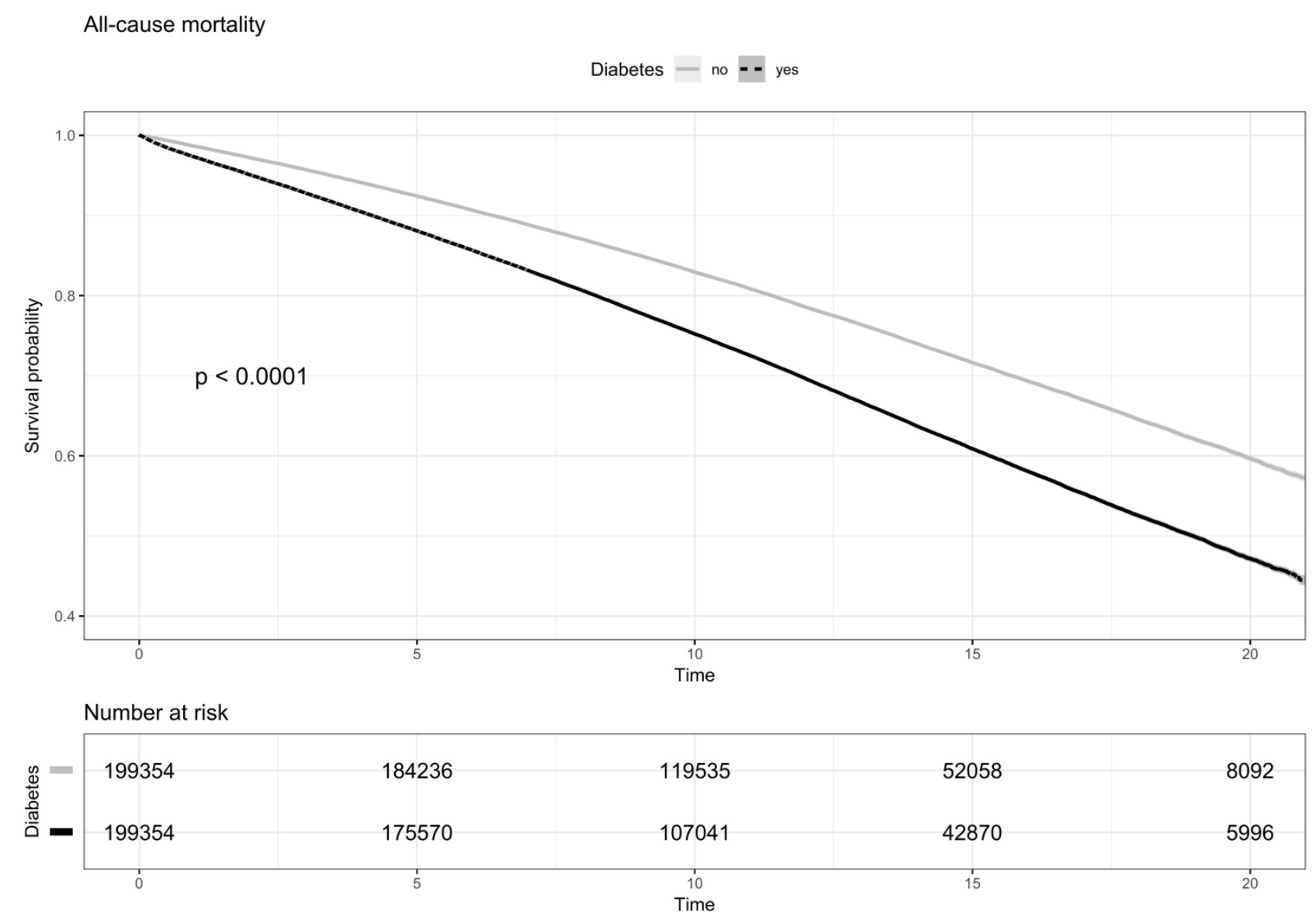

Appendix Figure 1. Survival after start of follow-up in diabetes groups. 

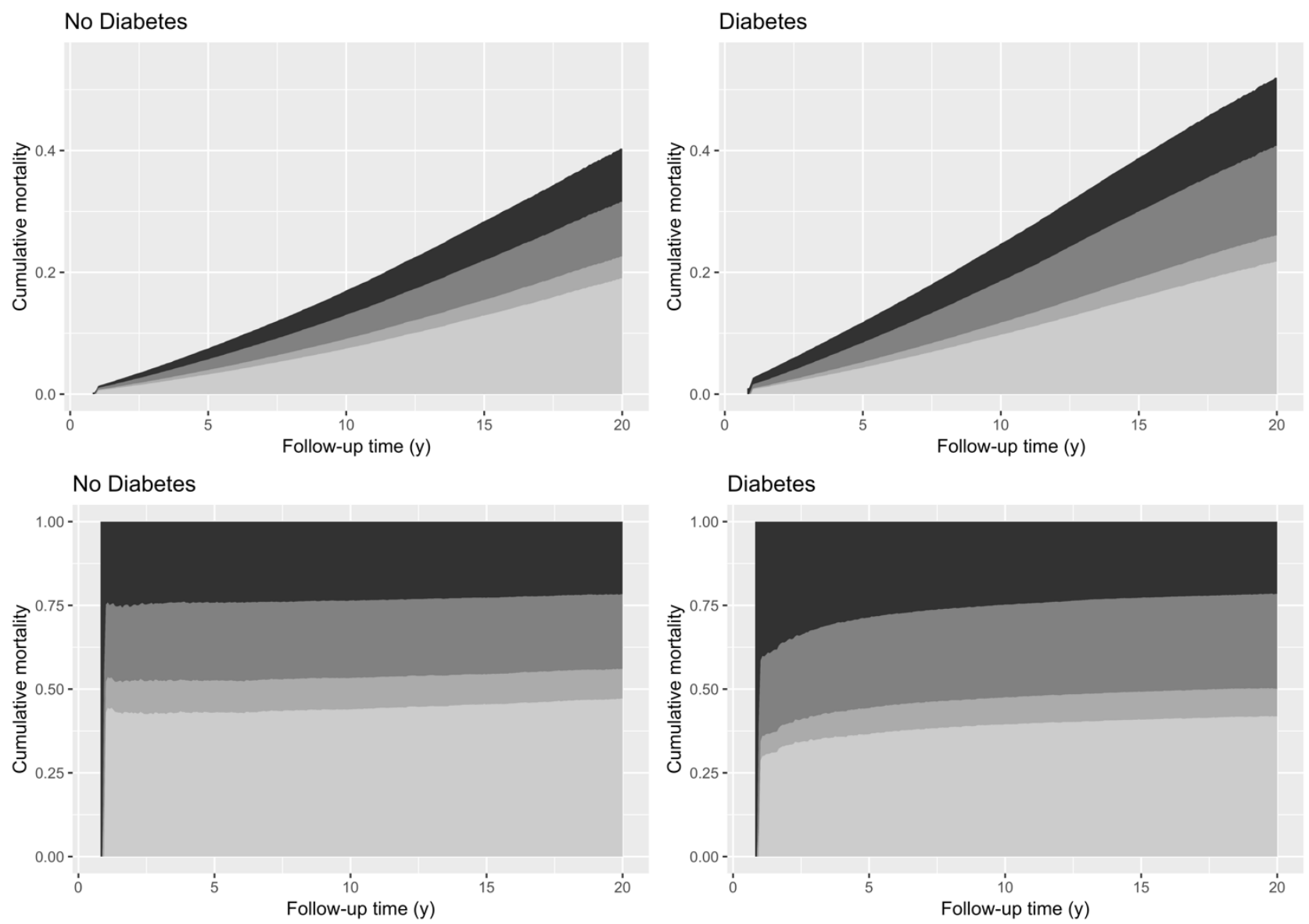

Appendix Figure 2. Cumulative mortality for causes of death, calculation started 0.5 years after the start of follow-up. Top actual values, bottom proportions of causes of death. Causes of death in shades of grey: other (light grey), stroke (grey), CHD (dark grey), cancer (black). 

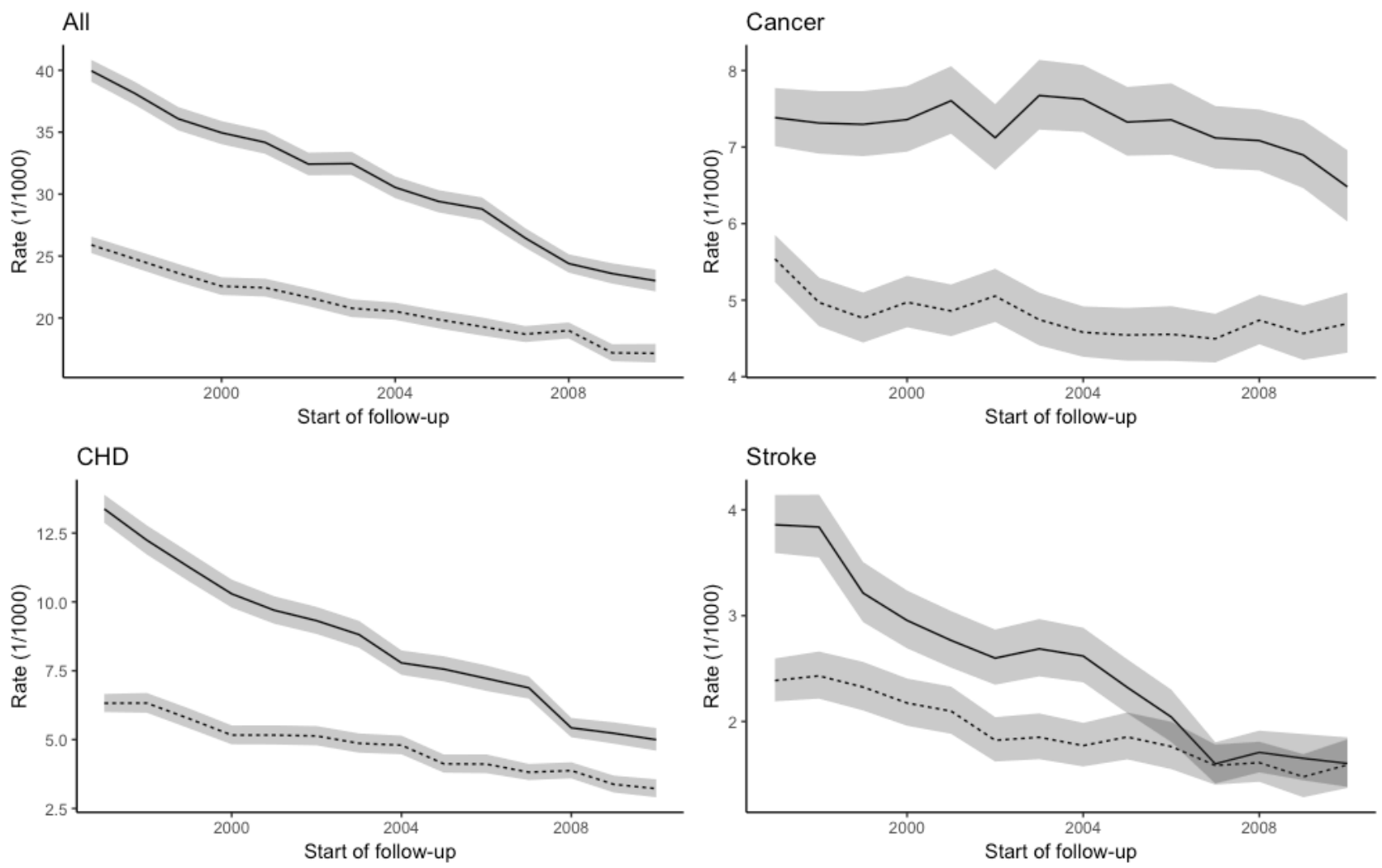

Appendix Figure 3. Unadjusted mortality rate (1000 person-years) with 95\% confidence intervals according to start of follow-up year for no DM (dashes) and DM (continuous line). 


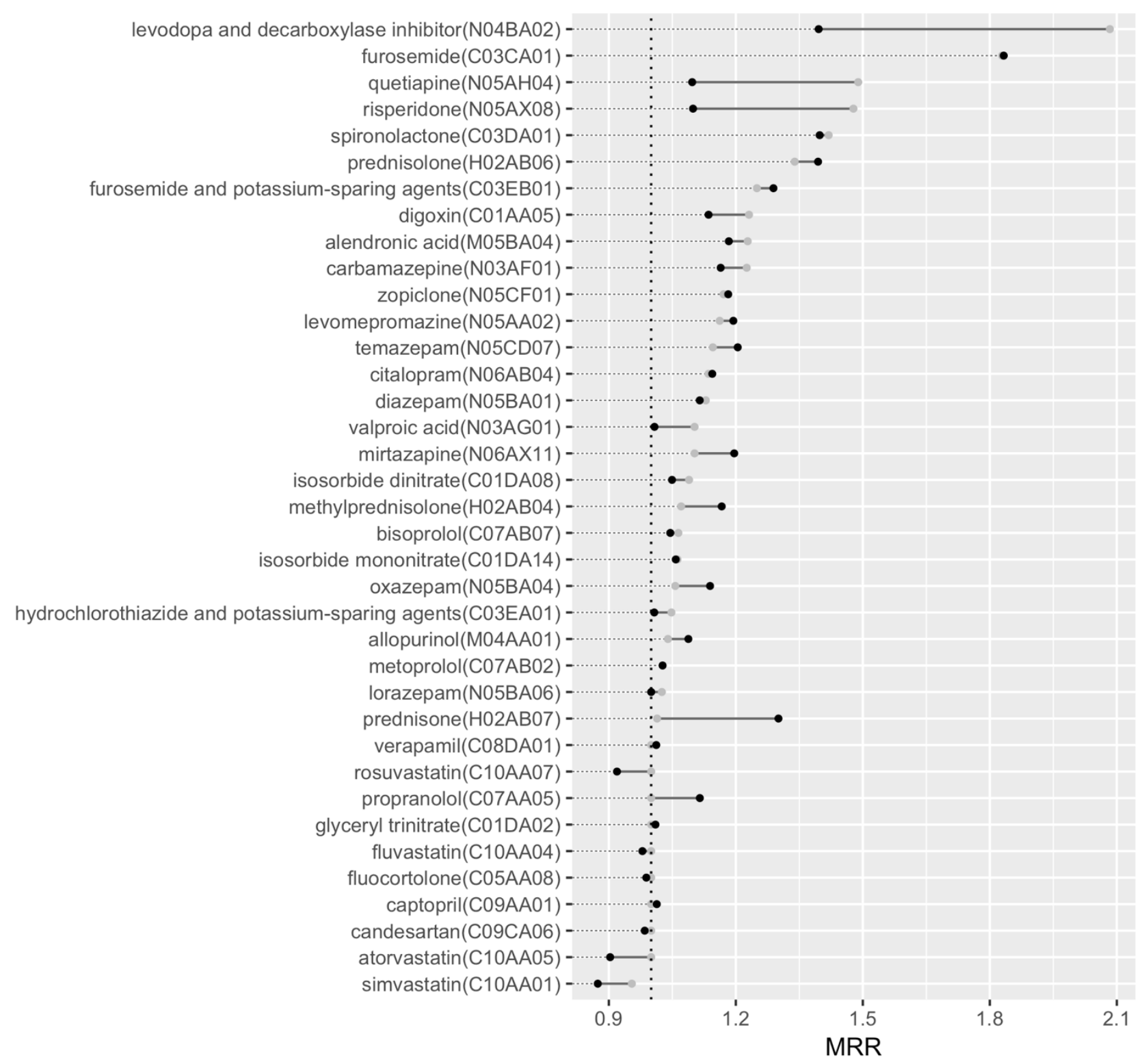

Appendix Figure 4. LASSO model for males. Mortality rate ratios for no DM (grey) and DM (black) populations. Models include age, sex, SOF year, and socio-economic group. A value of one (on vertical dashed line) means that variable was not included in the model. DM and no DM groups were modelled separately. 
levodopa and decarboxylase inhibitor(N04BA02) risperidone(N05AX08) furosemide(C03CA01) quetiapine(N05AH04) prednisolone(H02AB06) digoxin(C01AA05) levomepromazine(N05AA02) furosemide and potassium-sparing agents(C03EB01) citalopram(N06AB04) spironolactone(C03DA01) temazepam(N05CD07) -. prednisone(H02AB07) allopurinol(M04AA01) - alendronic acid(M05BA04) isosorbide dinitrate(C01DA08) isosorbide mononitrate(C01DA14) oxazepam(N05BA04) carbamazepine(N03AF01) enalapril(C09AA02) diazepam(N05BA01) perphenazine(N05AB03) - risedronic acid(M05BA07) zopiclone(N05CF01) valproic acid(N03AG01) rosuvastatin(C10AA07) mirtazapine(N06AX11) metoprolol(C07AB02) methylprednisolone(H02AB04) -· lorazepam(N05BA06) fluvastatin(C10AA04) bisoprolol(C07AB07) norethisterone and estrogen(G03FA01) atorvastatin(C10AA05) simvastatin(C10AA01) estradiol(G03CA03) -

$$
0.75
$$

$\ldots \ldots \ldots \ldots \ldots \ldots \ldots \ldots \ldots$

(4)

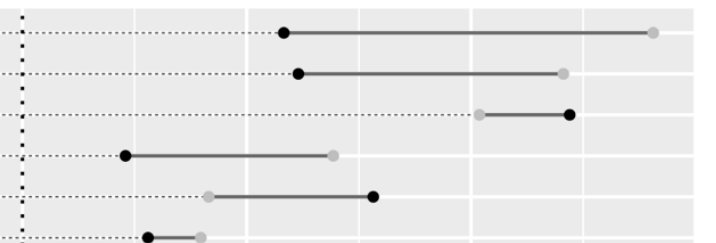

(2)

(1)
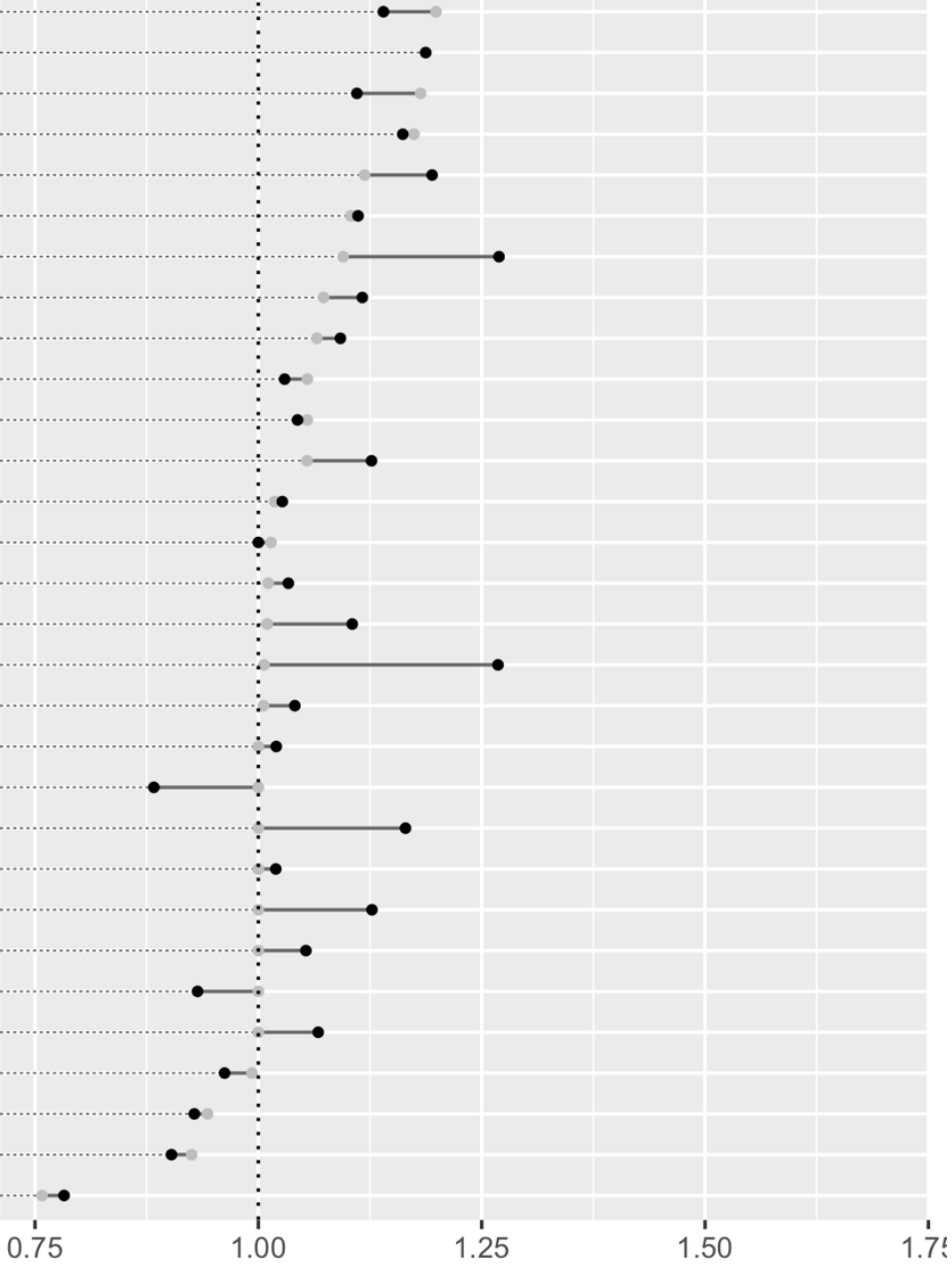

Females

Appendix Figure 5. LASSO model for females. Mortality rate ratios for no DM (grey) and DM (black) populations. Models include age, sex, SOF year, and socio-economic group. A value of one (on vertical dashed line) means that variable was not included in the model. DM and no DM groups were modelled separately. 
Appendix Table 1 Years of life lost in 15 years follow-up due to DM.

\begin{tabular}{|l|r|r|}
\hline Start of follow-up period & \multicolumn{1}{|l|}{ Male } & \multicolumn{1}{l|}{ Female } \\
\hline $1997-1999$ & 0.51 & 0.26 \\
\hline $2000-2001$ & 0.47 & 0.24 \\
\hline $2002-2003$ & 0.44 & 0.22 \\
\hline
\end{tabular}

